\title{
Cytoreductive Surgery versus Systemic Chemotherapy alone in Isolated Peritoneal Carcinomatosis of Colorectal Origin
}

\author{
Melike Ozcelik ${ }^{1}$, Abdlkerim Oyman, Ibrahim Cil ${ }^{1}$, Ozgul Duzgun², Omer Faruk Ozkan³ and Murat Ayhan ${ }^{3}$ \\ ${ }^{1}$ Department of Medical Oncology, University of Health Sciences, Umraniye Education and Research Hospital, Istanbul, Turkey \\ ${ }^{2}$ Department of General Surgery, University of Health Sciences, Umraniye Education and Research Hospital, Istanbul, Turkey \\ ${ }^{3}$ Department of Medical Oncology, Dr. Lutfi Kirdar, Kartal Training and Research Hospital, Istanbul, Turkey
}

\begin{abstract}
Objective: To compare the overall survival and progression-free survival of front-line cytoreductive surgery (CRs) \pm hyperthermic intraperitoneal chemotherapy versus intensive systemic chemotherapy alone, in patients with isolated peritoneal carcinomatosis of colorectal origin.

Study Design: Descriptive study.

Place and Duration of Study: Departments of Medical Oncology and Surgical Oncology in University of Health Sciences, Umraniye Education and Research Hospital, from January 2017 to January 2020.

Methodology: Clinicopathological data of patients presented with isolated peritoneal carcinomatosis were categorised into two groups according to their treatment type as patients who received intensive systemic chemotherapy alone or underwent front-line CRS \pm HIPEC. Overall and progression-free survival outcomes of the two approaches were quantified by survival analysis and compared with each other. The other collected variables were age, gender, performance status, tumor site and type of systemic chemotherapy.

Results: Overall, 109 patients were included. The median progression-free survival of patients treated with cytoreductive surgery \pm hyperthermic intraperitoneal chemotherapy was 12 months; whereas, it was 9 months in those treated with intensive systemic chemotherapy alone $(p=0.011)$. The median overall survival was estimated as 32 months in patients treated with cytoreductive surgery \pm hyperthermic intraperitoneal chemotherapy, compared with 23 months for those treated with systemic chemotherapy alone $(p=0.715)$.

Conclusion: Although not translated into overall survival gain, extended progression-free survival, may give an advantage to cytoreductive surgery \pm hyperthermic intraperitoneal chemotherapy when used with intensive systemic chemotherapy in the individualised treatment of isolated peritoneal carcinomatosis of colorectal carcinoma.
\end{abstract}

Key Words: Colorectal carcinoma, Cytoreductive surgery, Hyperthermic intraperitoneal chemotherapy, Overall survival, Peritoneal carcinomatosis, Systemic chemotherapy.

How to cite this article: Ozcelik M, Oyman A, Cil I, Duzgun O, Ozkan OF, Ayhan M. Cytoreductive Surgery versus Systemic Chemotherapy alone in Isolated Peritoneal Carcinomatosis of Colorectal Origin. J Coll Physicians Surg Pak 2021; 31(11):1308-1313.

\section{INTRODUCTION}

Peritoneal carcinomatosis is defined as the invasion of peritoneal surface by metastatic tumor deposits. Patients with peritoneal carcinomatosis arising from colorectal cancer generally have shorter survival compared with those without involvement of peritoneum. ${ }^{1}$

Correspondence to: Dr. Melike Ozcelik, Department of Medical Oncology, University of Health Sciences, Umraniye Education and Research Hospital, Turkey

E-mail:drmelike.ozcelik@gmail.com

Received: May 30, 2021; Revised: August 28, 2021;

Accepted: September 22, 2021

DOI: https://doi.org/10.29271/jcpsp.2021.11.1308
Historically, the goal of treatment was only palliative including palliative surgery intended usually for obstruction. While systemic chemotherapy represents a primary strategy exhibiting a clear survival advantage over best supportive care in the treatment, clinical outcome still remains poor. ${ }^{2-4}$

There is considerable interest in the implementation of cytoreductive surgery (CRS) \pm hyperthermic intraperitoneal chemotherapy (HIPEC), particularly as front-line therapy to improve survival outcomes in the peritoneal carcinomatosis setting arising from colorectal cancer. Cytoreductive surgery comprises surgical removal of visible tumor implants located most commonly in pelvis, bowel surfaces, subphrenic region, and lesser sac. The efficacy of this treatment approach is dependent on the amount of residual tumor deposits remaining after cytoreduction, which is estimated by using completeness of cytoreduction score. Persisting implants larger than $2.5 \mathrm{~cm}$ at 
the end of cytoreduction are an objective descriptor of suboptimal cytoreduction and correlate with poor survival. Although, early signs of clinical benefit from CRS were seen in various trials, $^{5-7}$ the advantage of this strategy has not been definitively demonstrated to date.

It was hypothesised that front-line CRS will result in better survival outcomes compared with intensive systemic chemotherapy alone, in isolated peritoneal carcinomatosis arising from colorectal cancer. There are several reasons front-line CRS could offer advantage over the standard systemic chemotherapy approach. Removal of invasive peritoneal implants aids in the reduction of disease burden. Because patients with peritoneal carcinomatosis have typically got more abdominal symptoms such as abdominal pain, intestinal obstruction, fistula formation, intractable nausea, vomiting and malnutrition, sustained quality of life is particularly important for these patients. ${ }^{8}$ Importantly, decline in quality of life poses a significant obstacle to the effective administration of systemic chemotherapy.

According to the current state of data available on colorectal cancer, there is controversy about the best course of action for the first line treatment of patients with peritoneal carcinomatosis. In a recent review of literature, one of the reasons of conflicting data on clinical efficacy of CRS, was reported to be the lack of randomised trials in the era of intensive systemic chemotherapy. ${ }^{9}$ It was argued that clinical benefit of CRS might have been much smaller if irinotecan, oxaliplatin, or molecularly targeted regimens were used as systemic therapy. The contribution of CRS \pm HIPEC to survival outcomes of colorectal cancer presented with isolated peritoneal carcinomatosis remains to be determined.

In this study, the aim was to compare the survival outcomes of front-line CRS \pm HIPEC followed by intensive systemic chemotherapy with intensive systemic chemotherapy alone in patients with isolated peritoneal carcinomatosis of colorectal origin.

\section{METHODOLOGY}

This retrospective decriptive study took place at the Departments of Medical Oncology and Surgical Oncology in the University of Health Sciences, Umraniye Education and Research Hospital. The clinicopathological data of patients diagnosed with advanced colorectal cancer presented with isolated peritoneal carcinomatosis between 01/01/2017 and 07/01/2020, were retrieved from preserved individual patient files in archive department or from hospital's database. Then, the medical records of patients who received intensive systemic chemotherapy alone or underwent front-line CRS \pm HIPEC were retrospectively reviewed by researchers. Following determination of suitable patients, the data were processed by one researcher to avoid bias. Parameters were assessed as per objective, including progression-free survival, overall survival, age, gender, performance status, tumor site and type of systemic chemotherapy. The overall and progression-free survival outcomes of the two above-mentioned treatment approaches for peritoneal carcinomatosis were quantified by survival analysis and compared with each other. Ethical approval was obtained from the Ethics Committee of University of Health Sciences, Umraniye Education and Research Hospital (Date: 08.10.2020, No. 331).

Inclusion criteria were: patients aged 18 years or older, pathologically proven colorectal adenocarcinoma, isolated peritoneal carcinomatosis, front-line CRS \pm HIPEC followed by intensive systemic chemotherapy or receipt of intensive systemic chemotherapy alone. Exclusion criteria were presence of any distant metastasis in addition to peritoneum (including limited number of liver metastases) poor performance status precluding the institution of intensive systemic chemotherapy regimen and suboptimal cytoreduction.

Intensive systemic chemotherapy regimen consisted of FOLFOX (Oxaliplatin $85 \mathrm{mg} / \mathrm{m}^{2}$ IV Day 1 , Leucovorin $400 \mathrm{mg} / \mathrm{m}^{2}$ IV Day 1, 5-FU $400 \mathrm{mg} / \mathrm{m}^{2}$ IV bolus on Day 1 then 1200 $\mathrm{mg} / \mathrm{m}^{2} /$ day $\times 2$ days IV continuous infusion) or FOLFIRI (Irinotekan 180 mg/m² IV over 30-90 min on Day 1, Leucovorin $400 \mathrm{mg} / \mathrm{m}^{2} \mathrm{IV}$ infusion to match duration of irinotecan infusion Day 1, 5-FU $400 \mathrm{mg} / \mathrm{m}^{2}$ IV bolus on Day 1, then $1200 \mathrm{mg} / \mathrm{m}^{2} /$ day x2 days IV continuous infusion) cycled every two weeks. Targeted monoclonal antibody and later line treatments provided after progression on first-line regimen were also recorded.

Data on cytoreductive surgery, which was performed by the same surgical team as described by Sugarbaker, ${ }^{10}$ were collected. HIPEC procedure consisted of the delivery of oxaliplatin (OXA; $300 \mathrm{mg} / \mathrm{m} 2 \mathrm{BSA}$ ) in $5 \%$ dextrose into the peritoneal cavity in $3 \mathrm{~L}$ of heated perfusion $\left(42^{\circ} \mathrm{C}-43^{\circ} \mathrm{C}\right)$ for $30 \mathrm{~min}$.

Patients were categorised into two groups, according to their treatment type for peritoneal carcinomatosis: patients who received intensive systemic chemotherapy alone or underwent front-line CRS \pm HIPEC. Comparison of patient and tumor characteristics between treatment groups including age, gender, performance status and tumor site was achieved using Chi-square test. Kaplan-Meier method was performed to estimate the survival curves. Log-rank test was used to compare survival times between treatment groups. Progression-free survival (PFS) was defined as the time from the date of diagnosis to the date of progression or death. Overall survival (OS) was defined as the time between the date of diagnosis and the date of death from any cause. Multivariate Cox proportional hazards analysis was done to determine the independent impact, if any, of the treatment type (intensive systemic chemotherapy alone vs. front-line CRS + HIPEC followed by systemic chemotherapy) along with age, gender, performance status, tumor localisation and type of systemic chemotherapy, on the overall survival. The statistical analyses were achieved by SPSS version 23.0 (IBM Corporation, Armonk, NY, US). p-value of $<0.05$ was accepted to indicate statistical significance. 
Table I: Patient and tumor characteristics according to treatment arm.

\begin{tabular}{|c|c|c|c|c|c|c|}
\hline & & \multicolumn{2}{|c|}{ CRS \pm HIPEC } & \multicolumn{2}{|c|}{ Intensive chemotherapy alone } & \multirow{2}{*}{$\mathrm{p}$} \\
\hline & & n \% & Median & n \% & & \\
\hline \multicolumn{2}{|l|}{ Age } & $59.1 \pm 11.8$ & 58 & $61.3 \pm 9.5$ & 60.0 & 0.623 \\
\hline \multirow{2}{*}{ Age } & $\leq 60$ & 26 & $53.1 \%$ & 29 & $48.3 \%$ & \multirow{2}{*}{0.623} \\
\hline & $>60$ & 23 & $46.9 \%$ & 31 & $51.7 \%$ & \\
\hline \multirow{2}{*}{ Gender } & Female & 19 & $38.8 \%$ & 19 & $31.7 \%$ & \multirow{2}{*}{0.438} \\
\hline & Male & 30 & $61.2 \%$ & 41 & $68.3 \%$ & \\
\hline \multirow{3}{*}{ ECOG performance status } & 0 & 26 & $53.1 \%$ & 34 & $56.7 \%$ & \multirow{2}{*}{0.078} \\
\hline & 1 & 19 & $38.8 \%$ & 36 & $43.3 \%$ & \\
\hline & 2 & 4 & $8.2 \%$ & 0 & $0.0 \%$ & \multirow{3}{*}{0.249} \\
\hline \multirow{2}{*}{ Tumour site } & Left colon & 30 & $61.2 \%$ & 43 & $71.7 \%$ & \\
\hline & Right colon & 19 & $38.8 \%$ & 17 & $28.3 \%$ & \\
\hline \multirow{2}{*}{ Systemic chemotherapy } & Oxaliplatin & 31 & $63.3 \%$ & 30 & $50.0 \%$ & \multirow{2}{*}{0.165} \\
\hline & Irinotecan & 18 & $36.7 \%$ & 30 & $50.0 \%$ & \\
\hline \multirow{2}{*}{ Targeted therapy } & Anti-VEGF & 28 & $57.1 \%$ & 37 & $61.7 \%$ & \multirow{2}{*}{0.632} \\
\hline & 1Anti-EGFR & 21 & $42.9 \%$ & 23 & $38.3 \%$ & \\
\hline
\end{tabular}

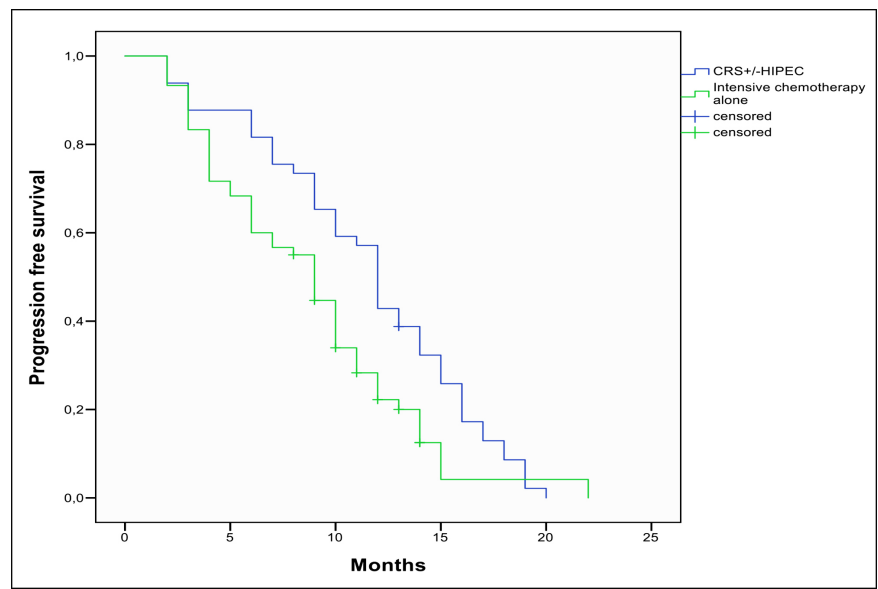

Figure 1: Progression-free survival curves of two treatment arms.

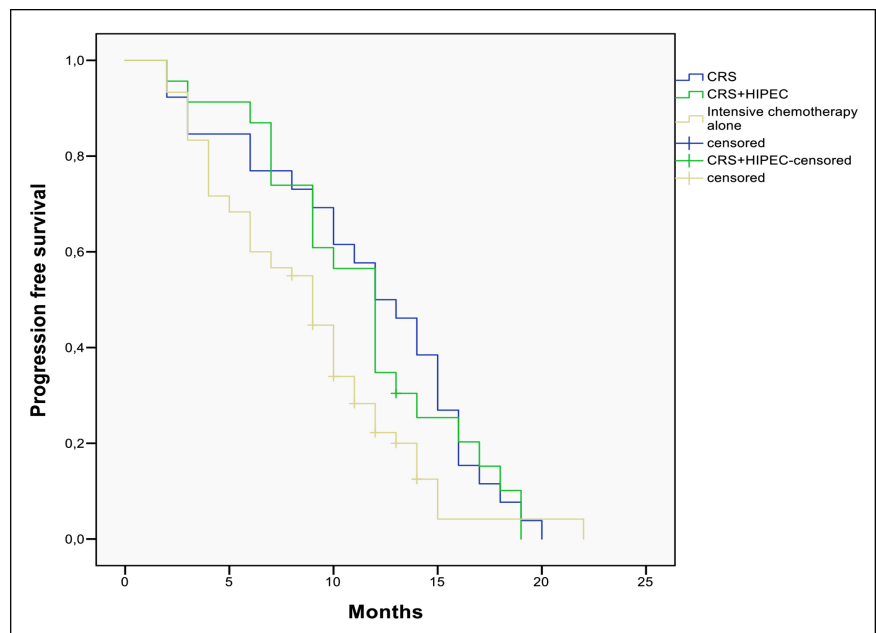

Figure 2: Progression-free survival curves of three treatment arms.

\section{RESULTS}

In total, 109 patients were included. Median age was 59 years, ranging from 27-77 years. There were 38 female (34.9\%) and 71 male patients (65.1\%). Sixty (55\%) out of 109 patients were started on intensive systemic chemotherapy alone as initial treatment choice, while 49 patients (45\%) underwent front-line CRS \pm HIPEC followed by intensive systemic chemotherapy. Among these 49 patients, 26 cases (53\%) underwent CRS without HIPEC due to technical inadequacy. The CRS \pm HIPEC and the intensive systemic chemotherapy alone groups were statistically similar in terms of clinical characteristics. Data about patient and tumor characteristics are presented in Table I.

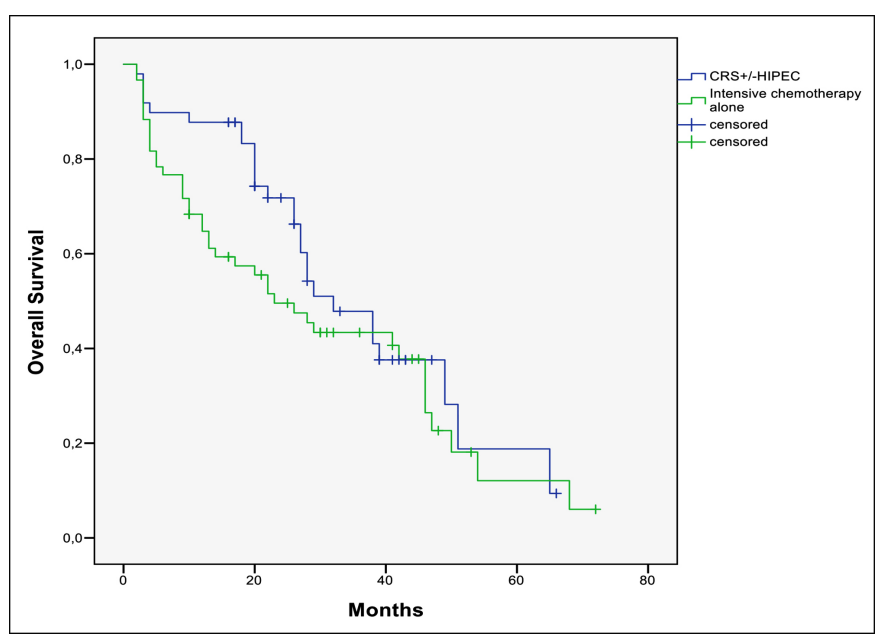

Figure 3: Overall survival curves of two treatment arms.

With regard to survival, median progression-free survival of all patients was found to be 10 (2-22) months. The median progression-free survival of patients treated with CRS \pm HIPEC followed by intensive systemic chemotherapy was 12 (11.0-12.9) months; whereas, it was 9 (7.7-10.2) months in those treated with intensive systemic chemotherapy alone $(p=0.011$, Figure 1$)$. When the groups were subdivided into three as CRS, CRS + HIPEC and intensive systemic chemotherapy alone; the median progression-free survival was similar between both CRS groups (either with or without HIPEC), whereas it was statistically significantly inferior in the intensive systemic chemotherapy alone group (Figure 2).

Median overall survival was $22(2-72)$ months for the entire study group. The median overall survival was estimated as 
32 (22-41) months in patients treated with CRS \pm HIPEC followed by systemic chemotherapy, compared with (13-32) 23 months in those treated with systemic chemotherapy alone ( $p=0.715$, Figure 3 ). Similarly, comparison of overall survival resulted in no statistically significant difference across CRS, CRS + HIPEC, and intensive systemic chemotherapy alone groups.

In the multivariate analysis, among the considered variables including age, gender, performance status, tumor site and treatment type, CRS \pm HIPEC was not identified to be an independent prognostic variable for improved overall survival $(p=0.332)$.

\section{DISCUSSION}

The entity of peritoneal carcinomatosis of colorectal origin, affecting approximately $10 \%$ of metastatic colorectal cancer cases, has still an unestablished treatment algorithm. In this study, we observed a significantly longer progression-free survival with front-line cytoreductive surgery (CRS) \pm hyperthermic intraperitoneal chemotherapy (HIPEC) than with first line intensive systemic chemotherapy alone, in patients with isolated colorectal peritoneal carcinomatosis. However, the overall survival gain with CRS \pm HIPEC did not meet the statistical criteria for significance.

Regarding systemic chemotherapy, the fluoropyrimidines alone were used for a long time in the history of metastatic colorectal cancer. Later, with the addition of newer chemotherapeutics and targeted therapy, all survival endpoints improved. Today, an intensive first-line systemic treatment regimen in metastatic colorectal cancer is composed of a combination of fluoropyrimidine, oxaliplatin/irinotecan, and a monoclonal antibody. ${ }^{11,12}$

Surgical management has also evolved in terms of the introduction of CRS \pm HIPEC in colorectal cancer involving peritoneal surface. Cytoreductive surgery combined with hyperthermic intraperitoneal chemoperfusion, after the well-established role in mesothelioma, pseudomyxoma peritonei, and some gynecological malignancies, has shown favorable duration of disease control in isolated peritoneal carcinomatosis of colorectal origin and an attempt has been made to ask for its benefit over systemic chemotherapy alone. ${ }^{6-8,13}$ Unfortunately, most trials comparing systemic chemotherapy alone with CRS \pm HIPEC found that the systemic chemotherapy group did not involve an intensive regimen or even the chemotherapeutics were not specified. ${ }^{14-18}$

Concerning the comparison of overall survival with literature, Mirnezami et al. in their research, reviewed 27 studies on CRS and intraperitoneal chemotherapy for colorectal peritoneal metastases. ${ }^{19}$ Most studies included in this review were in the form of case series and only four studies involved systemic chemotherapy as the control group. Among these four, two studies did not clearly describe which chemotherapeutics were used and in the other two, 5-Fluorouracil alone was reported to be utilised as systemic chemotherapy. The review concluded that improved overall survival can be achieved with the use of CRS and intraperitoneal chemotherapy. However, in the present study, 32 months of median overall survival were found in the CRS \pm HIPEC group, which was numerically superior to the 23 months (mo) of median overall survival were found in the intensive systemic chemotherapy alone group, but not statistically significant. This could be explained, by the fact that all the patients in the systemic chemotherapy alone group were administered intensive chemotherapy regimen including oxaliplatin or irinotecan in the current study. Again, either vascular endothelial growth factor (VEGF) or epidermal growth factor receptor (EGFR)-targeting therapies were used by all patients. Franko et al., with a very similar design to this study, compared the outcomes of CRS + HIPEC with systemic chemotherapy alone for colorectal peritoneal carcinomatosis. ${ }^{20}$ They found a statistically significant improvement in overall survival with CRS+HIPEC (34.7 mo. vs.16.8 mo, $p<0.001$ ). While the median overall survival times were very close to each other in CRS + HIPEC groups, the median overall survival of the systemic chemotherapy alone group in our study was superior to that of Franko et al. The most probable explanation is that, although not defined in detail, some patients completed their treatment course without oxaliplatin, irinotecan, or biological agents in that study. Besides, unlike our study, limited liver metastases and initial early-stage disease (a potential cause for later chemotherapy resistance due to previous usage of agents in adjuvant setting) were allowed in the two groups of the relevant study.

Concerning the comparison of progression-free survival with literature, results from a randomised trial involving 48 patients, half of whom received intensive systemic chemotherapy alone and the remaining half underwent front-line CRS+HIPEC, revealed that 5-year progression-free survival was $17 \%$ for the CRS+HIPEC arm compared with $0 \%$ for the intensive systemic chemotherapy alone arm of treatment. ${ }^{21}$ In the present study, progression-free survival (median of 12 months) was statistically significantly improved in favor of the CRS \pm HIPEC group which is consistent with the above-mentioned study with actuarial progression-free survival of 12 months in the surgery group.

With regard to HIPEC, this study also addressed the contribution of HIPEC to CRS. Hyperthermic intraperitoneal chemotherapy in addition to CRS did not show clear superiority over CRS alone and both progression-free survival and overall survival curves were similar for CRS with or without HIPEC. Recently, the multi-institutional PRODIGE 7 trial investigated the role for HIPEC in patients with isolated peritoneal carcinomatosis of colorectal cancer undergoing CRS. ${ }^{22}$ In line with our results, the authors did not find statistically significant difference among patients treated with or without 
HIPEC. This can be attributed to the use of multi-drug intensive systemic chemotherapy regimens, effectivity of which, together with targeted agents, is sufficient to omit the need for direct administration of chemotherapy into the peritoneal cavity.

Having an inherent selection bias due to the retrospective design is the most important limitation of the present study. Additionally, patient populations of treatment groups are relatively small, which may not permit for statistical significance in the analysis of some clinical variables.

\section{CONCLUSION}

There is no definite algorithm as to the best sequence of treatment options in isolated peritoneal carcinomatosis of colorectal carcinoma. According to the results of this study, the finding of improved progression-free survival, which is clinically and statistically validated along with the numerical increment in overall survival, demonstrates that CRS \pm HIPEC followed by intensive systemic chemotherapy is a valid treatment option in the modern era of chemotherapeutics.

\section{ETHICAL APPROVAL:}

This study was performed in line with the principles of the Declaration of Helsinki. Approval was obtained from the Ethics Committee of University of Health Sciences, Umraniye Education and Research Hospital (Date: 08.10.2020, No. 331).

\section{PATIENTS' CONSENT:}

Patients' consent was waived as the study design was retrospective.

\section{CONFLICT OF INTEREST:}

The authors declared no conflict of interest.

\section{AUTHORS' CONTRIBUTION:}

MO: Design, analyses, interpretation of work.

AO, OD: Revising, writing, acquistion.

IC: Writing review.

OFO: Drafting, review, acquistion of data.

MA: Acquistion of data review.

\section{REFERENCES}

1. Kranenburg O, van der Speeten $\mathrm{K}$, de Hingh I. Peritoneal metastases from colorectal cancer: Defining and addressing the challenges. Front Oncol 2021; 16 11: 650098. doi: 10.3389/fonc.2021.650098.

2. Venook AP, Niedzwiecki D, Lenz HJ, Innocenti F, Fruth B, Meyerhardt JA, et al. Effect of first-line chemotherapy combined with cetuximab or bevacizumab on overall survival in patients with KRAS wild-type advanced or metastatic colorectal cancer: A randomised clinical trial. Jama 2017; 317(23):2392-401. doi: 10.1001/jama.2017. 7105.
3. Heinemann V, von Weikersthal LF, Decker T, Kiani A, Kaiser F, Al-Batran SE, et al. FOLFIRI plus cetuximab or bevacizumab for advanced colorectal cancer: Final survival and per-protocol analysis of fire-3, a randomised clinical trial. $\mathrm{Br}$ J Cancer 2021; 124(3):587-94. doi: 10.1038/s41416-02001140-9.

4. Christina Wu. Systemic therapy for colon cancer. Surg Oncol Clin N Am 2018; 27(2):235-42. doi: 10.1016/j.soc.2017.11.001.

5. Nikolaos Vassos, Pompiliu Piso. Metastatic colorectal cancer to the peritoneum: Current treatment options. Curr Treat Options Oncol 2018 Sep 1;19(10):49. doi: 10.1007/ s11864-018-0563-8.

6. Huang CQ, Min Y, Wang SY, Yang XJ, Liu Y, Xiong B, et al. Cytoreductive surgery plus hyperthermic intraperitoneal chemotherapy improves survival for peritoneal carcinomatosis from colorectal cancer: a systematic review and meta-analysis of current evidence. Oncotarget 2017; 8(33): 55657-55683. doi: 10.18632/oncotarget.17497.

7. Horvath P, Yurttas C, Beckert S, Königsrainer A, Königsrainer I. Twelve-year single center experience shows safe Implementation of developed peritoneal surface malignancy treatment protocols for gastrointestinal and gynecological primary tumors. Cancers (Basel) 2021; 13(10):2471. doi: 10.3390/cancers13102471.

8. Ali G, Shaukat A, Masood S, Akram B, Ghaffar A, Gondal KM. A profile of colorectal tumors presenting as emergency. J Coll Physicians Surg Pak 2021; 31(1):74-78. doi: 10.29271/ jcpsp.2021.01.74.

9. Sánchez-Hidalgo JM, Rodríguez-Ortiz L, Arjona-Sánchez A, Rufián-Peña S, Casado-Adam A, Cosano-Álvarez A, et al. Colorectal peritoneal metastases: Optimal management review. World J Gastroenterol 2019; 25(27):3484-502. doi: 10.3748/wjg.v25.i27.3484.

10. Sugarbaker PH. Management of peritoneal metastases Basic concepts. J BUON 2015; 20:2-11.

11. Gustavsson B, Carlsson G, Machover D, Petrelli P, Roth A, $\mathrm{Schmoll} \mathrm{H}$, et al. A review of the evolution of systemic chemotherapy in the management of colorectal cancer. Clin Colorectal Cancer 2015; 14(1):1-10. doi: 10.1016/j.clcc.2014.11.002.

12. Marques RP, Duarte GS, Sterrantino C, Pais HL, Quintela A, Martins AP, et al. Triplet (FOLFOXIRI) versus doublet (FOLFOX or FOLFIRI) backbone chemotherapy as first-line treatment of metastatic colorectal cancer: A systematic review and meta-analysis. Crit Rev Oncol Hematol 2017; 118:54-62. doi: 10.1016/j.critrevonc.2017.08.006.

13. Bijelic L, Ramos I, Goeré D. The landmark series: Surgical treatment of colorectal cancer peritoneal metastases. Ann Surg Oncol 2021; 28(8):4140-4150. doi: 10.1245/ s10434-021-10049-3.

14. Repullo DJ, Barbois S, Leonard D, Bohlok A, Van den Audenaeren ET, Hendlisz A, et al. The absence of benefit of perioperative chemotherapy in initially resectable peritoneal metastases of colorectal cancer origin treated with complete cytoreductive surgery and hyperthermic intraperitoneal chemotherapy: A retrospective analysis. Eur J Surg Oncol 2021; 47(7):1661-7. doi: 10.1016/j.ejso.2021.01.018.

15. Witkamp AJ, de Bree E, Kaag MM, Boot $H$, Beijnen JH, van 
Slooten GW, et al. Extensive cytoreductive surgery followed by intra-operative hyperthermic intraperitoneal chemotherapy with mitomycin-C in patients with peritoneal carcinomatosis of colorectal origin. Eur J Cancer 2001; 37(8):979-84. doi: 10.1016/s0959-8049(01)00058-2.

16. Verwaal VJ, Boot $\mathrm{H}$, Aleman BM, van Tinteren $\mathrm{H}$, Zoetmulder FA. Recurrences after peritoneal carcinomatosis of colorectal origin treated by cytoreduction and hyperthermic intraperitoneal chemotherapy: Location, treatment, and outcome. Ann Surg Oncol 2004; 11(4):375-9. doi: 10.1245/ASO.2004.08.014.

17. Glehen O, Cotte E, Schreiber V, Sayag-Beaujard AC, Vignal J, Gilly FN. Intraperitoneal chemohyperthermia and attempted cytoreductive surgery in patients with peritoneal carcinomatosis of colorectal origin. Br J Surg 2004; 91(6):747-54. doi: 10.1002/bjs.4473.

18. Glehen O, Kwiatkowski F, Sugarbaker PH, Elias D, Levine EA, De Simone $M$, et al. Cytoreductive surgery combined with perioperative intraperitoneal chemotherapy for the management of peritoneal carcinomatosis from colorectal cancer: A multiinstitutional study. J Clin Oncol 2004; 22(16):3284-329. doi: 10.1200/JCO.2004.10.012.
19. Mirnezami R, Moran BJ, Harvey K, Cecil T, Chandrakumaran $\mathrm{K}$, Carr N, et al. Cytoreductive surgery and intraperitoneal chemotherapy for colorectal peritoneal metastases. World J Gastroenterol 2014; 20: 14018-32.

20. Franko J, Ibrahim Z, Gusani NJ, Holtzman MP, Bartlett DL, Zeh HJ. Cytoreductive surgery and hyperthermic intraperitoneal chemoperfusion versus systemic chemotherapy alone for colorectal peritoneal carcinomatosis. Cancer 2010; 116(16):3756-62. doi: 10.1002/cncr.25116.

21. Cashin PH, Mahteme H, Spång N, Syk I, Frödin KE, Torkzad $M$, et al. Cytoreductive surgery and intraperitoneal chemotherapy versus systemic chemotherapy for colorectal peritoneal metastases: A randomised trial. Eur J Cancer 2016; 53:155-62. doi: 10.1016/j.ejca.2015.09.017.

22. Quenet F, Elias D, Roca L, Goere D, Ghouti L, Pocard M, et al. Cytoreductive surgery plus hyperthermic intraperitoneal chemotherapy versus cytoreductive surgery alone for colorectal peritoneal metastases (PRODIGE 7): A multicentre, randomised, open-label, phase 3 trial. Lancet Oncol 2021; 22(2):256-66. doi: 10.1016/S1470-2045(20)30599-4. 